\title{
CONCENTRAÇÃO DE ESCÓRIA METALURGICA DA LIGA FESIMN POR MESA CONCENTRADORA WILFLEY
}

R. O. GALVÃO", F. B. M. BARROS, G. K. SILVA NETO, V. C. P. ANDRADE, E. L. SILVA, J. C. S. OLIVEIRA, B. B. LIRA

Universidade Federal do Ceará, Universidade Federal de Pernambuco.

engraulim@gmail.com*

Submetido 26/08/2017 - Aceito 13/09/2017

DOI: $10.15628 /$ holos.2017.6247

\section{RESUMO}

A produção industrial da liga metálica ferrosiliciomanganês (FeSiMn) está intimamente ligada ao processo pirometalúrgico. Porém, devido às limitações desse processo, observa-se um elevado percentual da liga FeSiMn nas pilhas de rejeito. Neste contexto, o objetivo deste trabalho foi escalonar uma rota de recuperação utilizando uma mesa oscilatória Wilfley. Para o estudo foi utilizada cerca de $40 \mathrm{~kg}$ de escória metalúrgica da liga FeSiMn. Analisaram-se as influências de 3 parâmetros fundamentais da mesa: granulometria $(<125 \mu \mathrm{m}$ e $>125 \mu \mathrm{m})$, inclinação $\left(2^{\circ}\right.$ e $\left.4^{\circ}\right)$ e amplitude (12 e $14 \mathrm{~mm}$ ). As influências dos parâmetros da mesa oscilatória foram analisadas pela variação do teor do concentrado, das recuperações mássica e metalúrgica. Para determinar o teor do concentrado empregou-se a técnica de fluorescência de raios-X (FRX). Com isso, constatou-se que os parâmetros amplitude e inclinação apresentaram uma menor influência sobre o teor e a recuperação mássica. No entanto, a granulometria apresentou uma variação significativa na recuperação metalúrgica. Assim, pode-se concluir que neste estudo a configuração que gerou uma maior eficiência no processo de concentração da liga FeSiMn, utilizando a mesa oscilatória Wilfley foi: granulometria $<125 \mu \mathrm{m}$; inclinação de $2^{\circ}$; e amplitude de $14 \mathrm{~mm}$. Essa configuração apresentou uma recuperação metalúrgica de aproximadamente $50 \%$ da liga FeSiMn.

PALAVRAS-CHAVE: Liga FeSiMn, Escoria, Mesa Oscilatória, FRX.

\section{CONCENTRATION OF FESIMn LEAGUE METALLURGICAL SLAG BY CONCENTRATOR TABLE WILFLEY}

\begin{abstract}
The industrial production of ferro-silico-manganese alloy (FeSiMn) is closely linked to the pyrometallurgical process. However, due to limitations in the process a high percentage of FeSiMn alloy is observed in the tailings piles. In this context, the purpose of this work is to stagger a recovery rotation of FeSiMn alloy by using a Wilfley oscillating table. A $40 \mathrm{~kg}$ slag sample from the FeSiMn alloy was utilized for the study. The influence of three essential parameters of the table were analyzed: granulometry $(<125 \mu \mathrm{m}$ and $>125 \mu \mathrm{m})$, slope $\left(2^{\circ}\right.$ and $\left.4^{\circ}\right)$ and amplitude (12 and $14 \mathrm{~mm})$. The influence of the oscillatory table's parameters was analyzed by the variation of the concentrate content and the mass and
\end{abstract}

metallurgical recoveries. The X-ray fluorescence (FRX) technique was used to determine the concentrate content. Hence, it was found that the amplitude and slope parameters had a lower influence on the content and recovery. However, the granulometry showed significant variation in metallurgical recovery. Thus, it can be concluded that in this study, the configuration that generated a higher efficiency in the concentration process of FeSiMn alloy using a Wilfley oscillating table was: granulometry $<125 \mu \mathrm{m} ; 2^{\circ}$ slope; and amplitude of $14 \mathrm{~mm}$. This configuration showed a metallurgical recovery of approximately $50 \%$ of the FeSiMn alloy.

KEYWORDS: FeSiMn League, Slag, Oscillatory Table, FRX. 


\section{INTRODUÇÃO}

O manganês é um elemento de transição da família do ferro e representa cerca de 0,1\%, em peso, dos constituintes do planeta (WILLS,1992). Depois do ouro e das pedras preciosas, foi o manganês um dos primeiros recursos minerais a despertar interesse no Brasil (ABREU, 1973). Como insumo mineral, tem um papel importante na indústria metalúrgica devido às suas características físico-químicas. Suas aplicações neste setor industrial estão vinculadas a produção de ferro-ligas, que são utilizadas para a fabricação do aço (GONÇALVES E SERFATY, 1976). Uma das ligas metálicas comumente utilizadas na produção de aço é a liga ferrosiliciomanganês (FeSiMn). Seu uso está associado a propriedade dessulfurante, e desoxidante, relacionada ao manganês e ao silício, respectivamente. Essas propriedades são fundamentais para a produção de aços mais resistentes (OLSEN E TANGSTAD, 2004). Isso mostra a importância do manganês no setor mineral e em suas aplicações nos produtos metalúrgicos.

Devido às limitações do processo metalúrgico, observa-se um elevado percentual da liga FeSiMn nas pilhas de rejeito (RICHTER, 2009; GALVÃO, 2016). A preocupação com os princípios de proteção do meio ambiente e a visão econômica de reprocessar materiais de baixo teor, tem gerado um maior aproveitamento desses rejeitos, tanto na mineração quanto nas siderúrgicas (REIS, 2005; GALVÃO, 2016). No entanto, para que seja possível o reprocessamento das pilhas de materiais de baixo teor é necessário aumentar a concentração do material, para isso é possível utilizar técnicas de concentração de materiais. Dentre as técnicas de concentração, a gravimétrica é comumente utilizada nessa etapa do processo. Essa técnica é baseada na diferença de densidade existente entre os materiais presentes, utilizando-se de um meio fluido (água ou ar) para que ocorra a efetiva separação. A aplicação adequada de processos de concentração nas plantas siderúrgicas poderá aumentar, potencialmente, o aproveitamento de seus rejeitos. Isso indica que a escolha do método de concentração a ser aplicado nas pilhas de rejeito metalúrgico deve satisfazer condições técnicas e econômicas ideais para a sua implantação (REIS, 2005). Com isso, fica claro a importância do estudo sobre técnicas de concentração para o reprocessamento de escoria metalúrgica.

Neste contexto, o objetivo deste trabalho foi determinar os parâmetros adequados de uma mesa oscilatória Wilfley, visando a maior concentração do material. Para o estudo foi utilizado escoria metalúrgica da liga FeSiMn proveniente da Empresa de Ferro Ligas Marabá. Analisou-se as influências de 3 parâmetros fundamentais da mesa oscilatória: granulometria $(<125 \mu \mathrm{m}$ e $>125 \mu \mathrm{m})$, inclinação $\left(2^{\circ}\right.$ e $\left.4^{\circ}\right)$ e amplitude (12 e $14 \mathrm{~mm}$ ). Para determinar a influência dos parâmetros da mesa oscilatória foi observado a variação do teor do concentrado e das recuperações mássica e metalúrgica. Para avaliar quantitativamente os teores do produto da concentração gravimétrica, empregou-se a técnica de fluorescência de raios $X$ (FRX). Com isso, observou-se que os 3 parâmetros analisados apresentaram influência nos teores de recuperação da liga FeSiMn. Pode-se concluir o melhor resultado, ou seja, a maior recuperação metalúrgica da liga, cerca de $53 \%$, foi observada utilizando a granulometria $<125 \mu \mathrm{m}$, a inclinação de $2^{\circ}$ e a amplitude de $14 \mathrm{~mm}$. 


\section{MATERIAL E MÉTODOS}

\subsection{Preparação e Caracterização da amostra}

Para este estudo, foi utilizada uma amostra de escória da liga FeSiMn de aproximadamente $40 \mathrm{~kg}$, proveniente da Empresa de Ferro Ligas Marabá. A amostra foi britada utilizando britadores de mandíbula e de rolos. O material britado foi classificado entre $2 \mathrm{~mm}$ (10 malhas). O produto dessa britagem foi homogeneizado e quarteado em amostras de $4 \mathrm{~kg}$. Essas amostras foram novamente quarteadas em alíquotas de $500 \mathrm{~g}$, as quais foram moídas utilizando um moinho de disco. O produto da moagem foi classificado em $115 \mu \mathrm{m}$ (120 malhas), gerando 2 alíquotas ( $<125 \mu \mathrm{m}$ e $>125 \mu \mathrm{m})$.

Realizou-se uma caracterização previa das amostras empregando a técnica de microscopia eletrônica de varredura (MEV), analisada por energia dispersiva de raios-X (EDS Energy Dispersal Spectroscopy). A Figura 1 apresenta o espectro obtido pelo EDS da liga FeSiMn e uma imagem microscópica (ES) da partícula da amostra. Foi observado a presença dos elementos: Ferro (Fe); Alumínio (Al); Manganês (Mn); e Silício (Si). Isso comprova a presença da liga FeSiMn na amostra estudada.

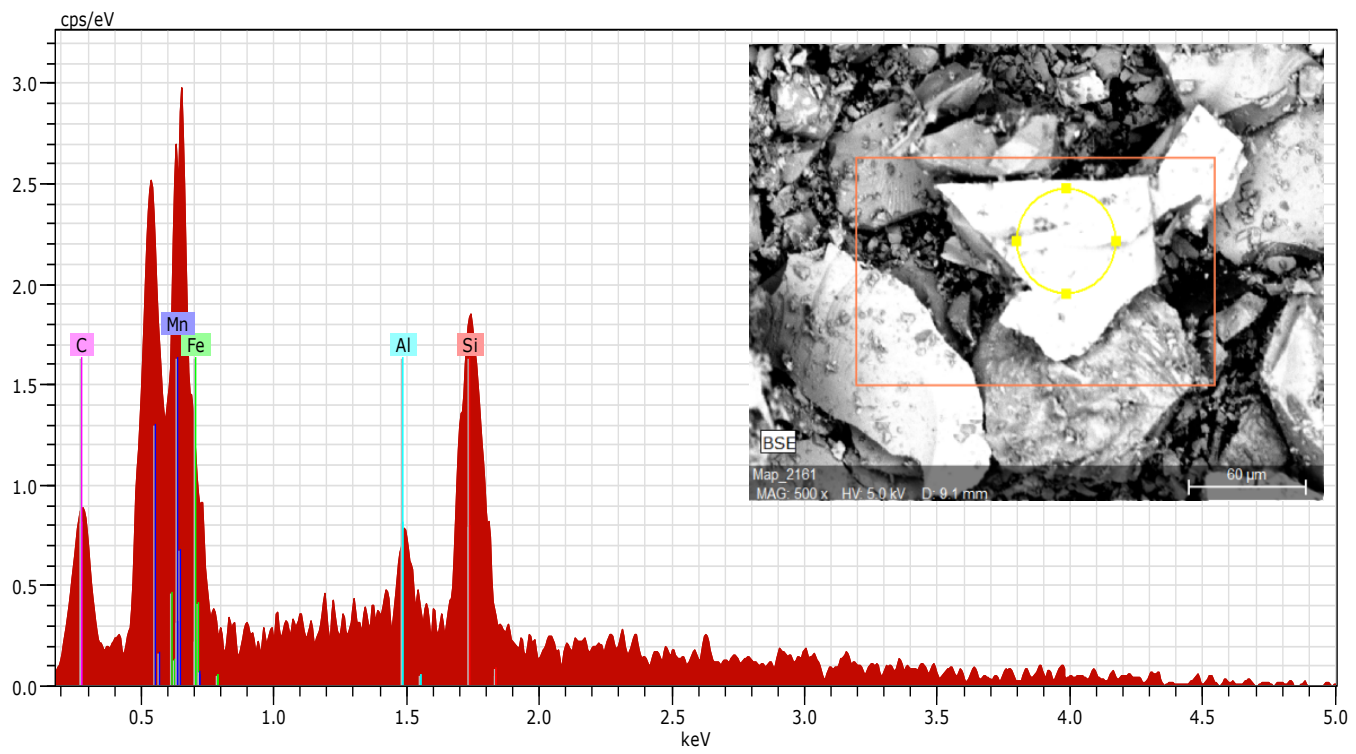

Figura 1: Espectro EDS da análise pontual na partícula da liga FeSiMn e uma imagem morfológica da partícula da liga, por elétrons secundários.

\subsection{Concentração Gravimétrica}

Para a etapa da concentração gravimétrica utilizou-se uma mesa oscilatória, do tipo Wilfley. Foi utilizado cerca de $250 \mathrm{~g}$ de amostra em cada ensaio. Neste estudo foram avaliados 3 parâmetros principais do funcionamento da mesa, os quais são vinculados a eficiência do processo de concentração: (i) Granulometria; (ii) Amplitude; e (iii) Inclinação. Para cada parâmetro foi empregado dois valores distintos. A Tabela 1 apresenta as configurações empregadas na mesa em cada ensaio. Alguns parâmetros da mesa foram fixados: fluxo de água $60 \mathrm{~L} / \mathrm{h}$; frequência de $360 \mathrm{rpm}$; e taxa de alimentação $1 \mathrm{~kg} / \mathrm{h}$. A amostra de escória foi descarregada na mesa em forma de polpa e de forma manual. Utilizando bandejas de aço, coletou-se os produtos da mesa oscilatória, denominadas de concentrado (porção com maior 
concentração da liga) e de leve (rejeito). Cabe ressaltar que os ensaios da mesa oscilatória foram realizados em duplicata, para garantir a reprodutibilidade dos ensaios. Os resultados obtidos no processo de concentração da liga de FeSiMn presente na escória foram analisados percentualmente por recuperação mássica e metalúrgica e pelo teor do concentrado.

Tabela 1: Parâmetros utilizados nos testes de concentração em mesa oscilatória Wilfley.

\begin{tabular}{l|l|l|l}
\hline Ensaios & Granulometria( $\boldsymbol{\mu m})$ & Inclinação( & Amplitude(mm) \\
\hline 1 & $<125$ & 2 & 12 \\
\hline 2 & $>125$ & 2 & 12 \\
\hline 3 & $<125$ & 4 & 12 \\
\hline 4 & $>125$ & 4 & 12 \\
\hline 5 & $<125$ & 2 & 14 \\
\hline 6 & $>125$ & 2 & 14 \\
\hline 7 & $<125$ & 4 & 14 \\
\hline 8 & $>125$ & 4 & 14 \\
\hline
\end{tabular}

\subsection{Fluorescência de raios-X}

Foi empregado o FRX para analisar, de forma quantitativa, a composição das amostras de alimentação da mesa oscilatória e das faixas concentradas do produto de cada ensaio. Cada amostra foi adequada a análise, ou seja, sua granulometria foi reduzida a um tamanho de partícula inferior a $75 \mu \mathrm{m}$, por meio de almofariz e pistilo.

Uma alíquota de cada amostra foi secada em estufa a $110^{\circ} \mathrm{C}$. Uma porção da alíquota seca foi levada a uma mufla a $1000^{\circ} \mathrm{C}$ por 2 horas para determinação da perda ao fogo. A outra porção da alíquota foi prensada em cápsulas de alumínio aplicando 30 toneladas de força. As pastilhas prensadas foram analisadas em espectrômetro de fluorescência de raio-X Rigaku Modelo ZsX Primus II, equipado com tubo de Rh e 7 cristais analisadores. A Tabela 1 apresenta os dados obtidos pela análise FRX.

Tabela 2. Percentual de óxidos constituintes das amostras de alimentação determinado por espectrometria FRX após perda ao fogo a $1000^{\circ} \mathrm{C}$.

\begin{tabular}{l|l|l|l|l|l|l|l|l|l}
\hline Alimentação & $\mathrm{Na}_{2} \mathrm{O}$ & $\mathrm{Al}_{2} \mathrm{O}_{3}$ & $\mathrm{SiO}_{2}$ & $\mathrm{P}_{2} \mathrm{O}_{5}$ & $\mathrm{~K}_{2} \mathrm{O}$ & $\mathrm{CaO}$ & $\mathrm{MnO}$ & $\mathrm{Fe}_{2} \mathrm{O}_{3}$ total & $\mathrm{BaO}$ \\
\hline$<125 \mu \mathrm{m}$ & 0.07 & 9.45 & $\mathbf{2 2 . 3 4}$ & 0.02 & 4.33 & 1.12 & $\mathbf{5 3 . 0 5}$ & $\mathbf{2 . 5 3}$ & 5.37 \\
\hline$>125 \mu \mathrm{m}$ & 0.06 & 9.63 & $\mathbf{2 2 . 7 3}$ & 0.01 & 4.42 & 1.1 & $\mathbf{5 2 . 6 8}$ & $\mathbf{1 . 9 1}$ & 5.64 \\
\hline
\end{tabular}




\section{RESULTADOS E DISCUSSÃO}

A Figura 2 exibe os resultados dos testes de concentração da liga metálica na mesa oscilatória, contendo dados sobre a recuperação média em massa e a recuperação metalúrgica e o teor médio de Fe presente. Para os ensaios 1 e 5 foram obtidos elevados teores de ferro com altas taxas de recuperação em massa, enquanto que menores teores foram obtidos nos ensaios 3 e 7. Na análise do balanço metalúrgico as maiores recuperações ocorreram nos ensaios 5 e 6 e de forma contraria para os ensaios 2 e 8 .

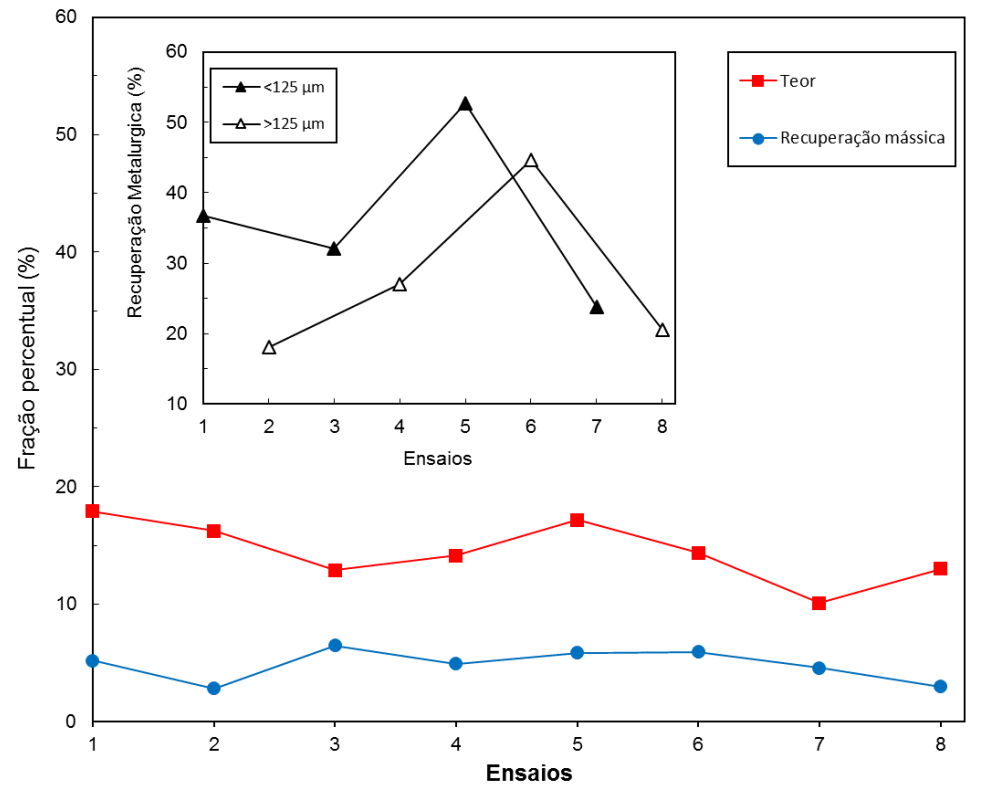

Figura 2: Gráfico da recuperação em massa versus recuperação metalúrgica

As maiores taxas de recuperação da liga metálica estiveram relacionadas com menores inclinações da mesa oscilatória, alcançando recuperações metalúrgicas de 52 e 44,66\%, para os ensaios 5 e 6, respectivamente. Estes dados corroboram com os trabalhos realizados por Reis (2005) que estudou a recuperação de minérios de manganês em mesa oscilatória em ângulos de $3^{\circ}$ e $5^{\circ}$. Os resultados obtidos foram de maior recuperação em todas as faixas granulométricas estudadas (global, $+0,074 \mathrm{~mm}$ e $-0,074 \mathrm{~mm}$ ) para o ângulo de inclinação em $3^{\circ}$. Para o ensaio 2 executado em inclinação de $2^{\circ}$ a baixa recuperação se deu em função do estreitamento entre as faixas de concentrado e rejeito provocadas pela baixa aceleração longitudinal resultado da menor amplitude (12 $\mathrm{mm})$.

As Figuras 3, 4 e 5 mostram o perfil de distribuição das partículas sobre a mesa permitindo avaliar o efeito das variáveis granulometria, inclinação e amplitude, respectivamente. $\mathrm{Na}$ Figura 3 os ensaios com inclinação em $2^{\circ}$ e amplitude de $12 \mathrm{~mm}$ tiveram redução na recuperação metalúrgica: de $36,74 \%$ para $18,05 \%$ ao mudar de granulometria fina para granulometria grossa. 

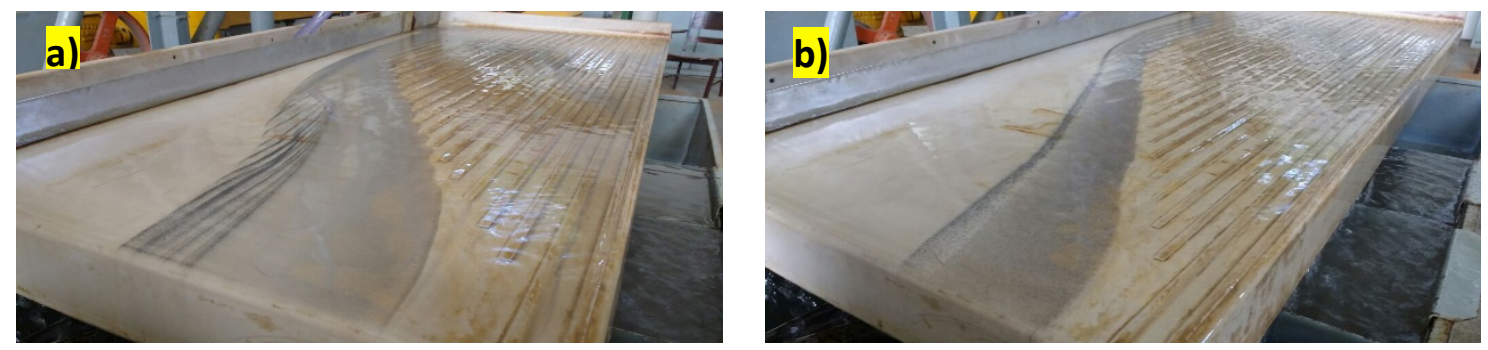

Figura 3: Perfil de separação no ensaio 1 (a) e ensaio 2 (b) para as condições de granulometria fina e granulometria grossa

Na Figura 4 os ensaios com granulometria fina e amplitude de $14 \mathrm{~mm}$ tiveram diminuição na recuperação metalúrgica: de $52,64 \%$ para $23,81 \%$ ao variar de uma inclinação de $2^{\circ}$ para $4^{\circ}$.
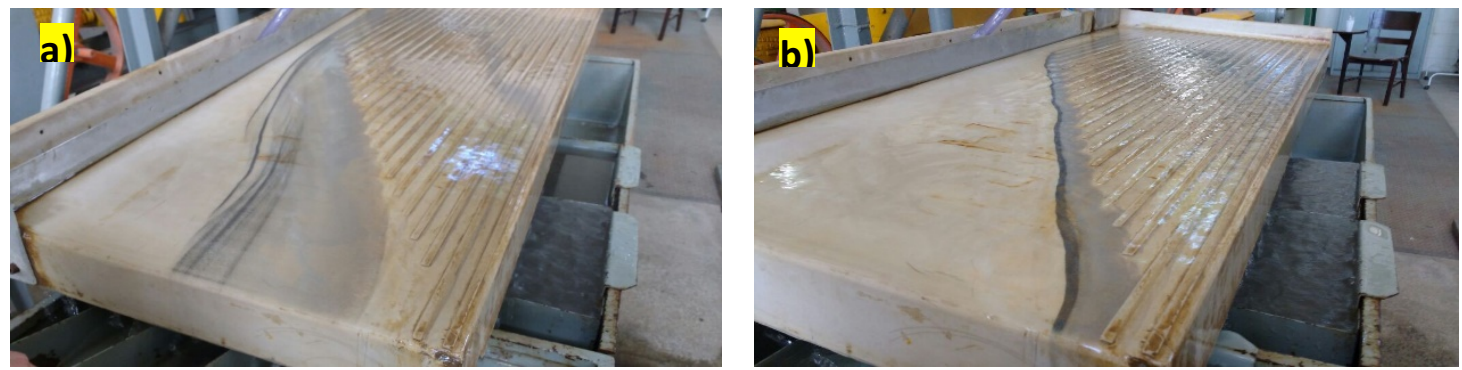

Figura 4: Perfil de separação no ensaio 5 (a) e ensaio 7 (b) para as condições de inclinação $2^{\circ}$ e $4^{\circ}$, respectivamente

Na Figura 5 os ensaios com granulometria grossa e inclinação em $4^{\circ}$ tiveram diminuição na recuperação metalúrgica: de 27,07\% para 20,53 \% ao variar de uma amplitude em $12 \mathrm{~mm}$ para $14 \mathrm{~mm}$. Interessante destacar, que para os ensaios 1 e 5, em que ocorreram em granulometria fina e inclinação de $2^{\circ}$, o aumento da amplitude promove maior recuperação metalúrgica, de $36 \%$ para $52 \%$, respectivamente.
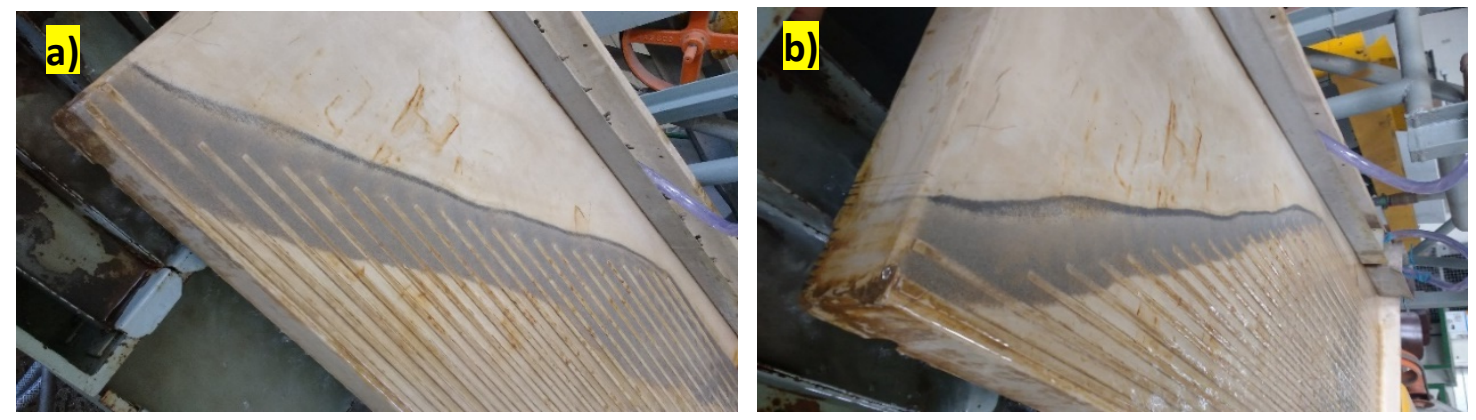

Figura 5: Perfil de separação no ensaio 4 (a) e ensaio 8 (b) para as condições de amplitude $12 \mathrm{~mm}$ e $14 \mathrm{~mm}$, respectivamente

Este efeito oposto obtido nos ensaios 1 e 5 nos permite afirmar que em menores inclinações e granulometrias mais finas as partículas densas são as últimas a sofrer o efeito do filme líquido, em contrapartida são as primeiras a sofrer efeito com o aumento do movimento oscilatório, justificando assim a boa recuperação metalúrgica no ensaio 5 . De modo contrário utilizando maior inclinação o efeito no escorregamento é maior para as partículas grossas densas, 
quando comparadas com as finas densas, sendo o contrário no movimento oscilatório com menor efeito nas grossas densas do que nas finas densas. Em síntese a explanação acima obtida a partir da análise dos trabalhos de Burt (1984) permitem compreender a menor recuperação metalúrgica para o ensaio 8.

\section{CONCLUSÕES}

O processo de beneficiamento gravimétrico por mesa oscilatória se mostrou eficiente para a concentração da liga metálica de FeSiMn. Destacam-se que as três variáveis estudadas foram significativas na recuperação metalúrgica. Observou-se que os ensaios com granulometria grossa e fina em amplitudes de $14 \mathrm{~mm}$ e inclinação de $2^{\circ}$ promoveram recuperações metalúrgicas de $52 \%$ e $44,66 \%$, respectivamente.

Em termos gerais, os resultados dos testes de concentração em mesa oscilatória mostraram que a maior recuperação metalúrgica da liga de FeSiMn, ocorre com a variável de inclinação em $2^{\circ}$ e amplitudes mais elevadas.

\section{AGRADECIMENTOS}

Os autores agradecem ao laboratório de tratamento de minérios (LTM) da Universidade Federal de Pernambuco pela disponibilidade do espaço para a realização dos ensaios e à empresa Ferro Ligas Marabá (FERMAR) pela doação da escória para o estudo.

\section{REFERÊNCIAS}

ABREU, S.F (1973). Recursos minerais do Brasil. São Paulo, SP, Editora Edgard Blucher LTDA, vol.2, Cap.14, p.492-550.

BURT, R.O.; MILLS, C. Gravity Concentration Technology (Developments in Mineral Processing). Elsevier: Volume 5. The Netherlands. Amsterdam 1984.

GONÇALVES, E.; SERFATY, A (1976). Perfil Analítico do Manganês. Departamento Nacional da Produção Mineral, DNPM, Brasília, DF, Boletim $\mathrm{n}^{\circ}$ $37,149 \mathrm{p}$.

GALVÃO, R. O. Aplicação de um planejamento fatorial na recuperação de liga metálica (FeSiMn) de escória empregando-se mesa oscilatória do tipo wilfley. Dissertação de mestrado, Universidade Federal de Pernambuco (UFPE), Recife, 2016.

OLSEN, S. E. e TANGSTAD, M. Silicomanganese production and process understanding. Tenth International Ferroalloys Congress. Capetown, África do Sul, 2004.

REIS, E. L. Caracterização de resíduos provenientes da planta de beneficiamento do minério de manganês sílico carbonatado da RDM-Unidade Morro da Mina. Dissertação mestrado, Universidade Federal de Ouro Preto, Ouro Preto, 2005.

RICHTER, D. Uma rota de recuparação de metal a partir de escória secundária da produção de ferroníquel. Dissertação de mestrado, Universidade de São Paulo (USP), São Paulo, 2006.

WILLS, B. A (1992). Mineral processing Technology. Pergamon Press, 5a edição, New York, 855 p. 